D.E. BLAIR, G. D. LUDDEN AND K. YANO

KŌDAI MATH. SEM. REP.

27 (1976), 313-319

\title{
SEMI-INVARIANT IMMERSIONS
}

\author{
By David E. Blair*, Gerald D. Ludden* and Kentaro Yano
}

\section{$\S 1$. Introduction.}

Let $\tilde{M}$ be a differentiable manifold and let $\tilde{F}$ be a tensor field of type $(1,1)$ defined on $\tilde{M}$. If $M$ is a submanifold immersed in $\tilde{M}, M$ is said to be an invariant submanifold if the tangent space to $M$ at each point of $M$ is invariant under the endomorphism $\tilde{F}$. If $\tilde{M}$ is a complex manifold and $\tilde{F}$ is the almost complex structure on $\tilde{M}$ then the invariant submanifolds of $\tilde{M}$ are just the complex submanifolds. (See, Schouten and Yano [4]). If $\tilde{M}$ is a normal contact (or Sasakian) manifold with $(\tilde{F}, \tilde{\xi}, \tilde{\eta})$ as the almost contact structure on $\tilde{M}$, then there does not exist an invariant submanifold $M$ with $\tilde{\xi}$ normal to $M$. (See $\S 4$ ). Invariant submanifolds have been studied by many people. (See, Kubo [2], Schouten and Yano [4], Yano and Okumura [9], [10]).

The purpose of this paper is to study submanifolds $M$ of $\tilde{M}$ for which there is a distribution $D$ that is nowhere tangent to $M$ and such that the subspace spanned by $D$ and the tangent space to $M$ is invariant under $\widetilde{F}$ and $\widetilde{F} D$ is tangent to $M$ at each point of $M$. (See, Tashiro [6]).

\section{$\S 2$. Preliminaries.}

Let $M$ be a differentiable manifold. A tensor field $F$ of type $(1,1)$ on $M$ defines an almost complex structure if

$$
F^{2}=-I \text {, }
$$

in which case $M$ is of even dimension. This almost complex structure is integrable, i. e., $M$ is complex, if $[F, F]=0$, where $[F, F]$ is the Nijenhuis tensor of $F$ defined by

$$
[F, F](X, Y)=[F X, F Y]-F[F X, Y]-F[X, F Y]+F^{2}[X, Y] .
$$

Here $X$ and $Y$ are vector fields on $M$. A Riemannian metric $g$ is a Hermitian metric for $F$ if

$$
g(F X, F Y)=g(X, Y) \text {. }
$$

Received June 24, 1974.

* Work done under partial support by NSF Grant No. 36684 . 
An almost contact structure on $M$ is defined by

$$
\phi^{2}=-I+\eta \otimes \xi, \quad \phi \xi=0, \quad \eta \circ \phi=0, \quad \eta(\xi)=1,
$$

where $\phi$ is a tensor field of type $(1,1), \xi$ is a vector field and $\eta$ is a 1 -form on $M$. (See, Sasaki [3]). $M$ is of odd dimension and this almost contact structure is said to be normal if $[\phi, \phi]+d \eta \otimes \xi=0$. A Riemannian metric $g$ on $M$ is associated with the almost contact structure on $M$ if

$$
g(\phi X, \phi Y)=g(X, Y)-\eta(X) \eta(Y), \quad \eta(X)=g(\xi, X) .
$$

In this case we say that $M$ has an almost contact metric structure.

We say $M$ has an $(f, U, V, u, v, \lambda)$-structure if

$$
\begin{gathered}
f^{2}=-I+u \otimes U+v \otimes V, \\
f U=-\lambda V, \quad f V=\lambda U, \quad u \circ f=\lambda v, \quad v \circ f=-\lambda u, \\
u(U)=v(V)=1-\lambda^{2}, \quad u(V)=v(U)=0,
\end{gathered}
$$

where $f$ is a tensor field of type $(1,1), U$ and $V$ are vector fields, $u$ and $v$ are 1 -forms and $\lambda$ is a function on $M . M$ is of even dimension and this $(f, U, V$, $u, v, \lambda$ )-structure is normal if $[f, f]+d u \otimes U+d v \otimes V=0$. A Riemannian metric $g$ on $M$ is associated with this structure if

$$
\begin{gathered}
g(f X, f Y)=g(X, Y)-u(X) u(Y)-v(X) v(Y), \\
u(X)=g(U, X), \quad v(X)=g(V, X)
\end{gathered}
$$

and we say that $M$ has a $(f, g, u, v, \lambda)$-structure. (See, Blair, Ludden and Yano [1], Yano and Okumura [7], [8]).

\section{§3. Semi-invariant submanifolds of almost complex manifolds.}

Let $\tilde{M}$ be an almost complex manifold with almost complex structure $\tilde{F}$ and let $\tilde{U}$ be a non-vanishing vector field on $\tilde{M}$. Let $\iota: M \rightarrow \tilde{M}$ be an immersion such that

$$
\left\{\begin{array}{l}
\tilde{F} \iota_{*} X=\iota_{*} f X-\eta(X) \tilde{U}, \\
\tilde{F} \tilde{U}=\iota_{*} \xi, \\
\tilde{U} \text { is never tangent to } \iota(M)
\end{array}\right.
$$

for all vector fields $X$ on $M$, where $\iota_{*}$ is the differential of the immersion. Then we say that $M$ is a semi-invariant submanifold of $\tilde{M}$ with respect to $\tilde{U}$. Thus $f, \xi, \eta$ are a tensor field of type $(1,1)$, a vector field and a 1 -form on $M$ respectively. A straightforward calculation shows that

$$
f^{2}=-I+\eta \otimes \xi, \quad f \xi=0, \quad \eta \circ f=0, \quad \eta(\xi)=1,
$$


that is, $(f, \xi, \eta)$ is an almost contact structure on $M$. Thus we have the following theorem.

THEOREM 3.1. If $\tilde{M}$ is an almost complex manifold and $\tilde{U}$ is a non-vanıshing vector field on $\tilde{M}$, then a semi-invariant submanifold $M$ of $\tilde{M}$ with respect to $\tilde{U}$ possesses an induced almost contact structure.

If $\tilde{M}$ is complex, then we have that

$$
\begin{aligned}
0= & {[\tilde{F}, \tilde{F}]\left(\iota_{*} X, \iota_{*} Y\right) } \\
= & \iota_{*}\{[f, f](X, Y)+d \eta(X, Y) \xi\} \\
& -\left\{\left(\mathcal{L}_{f X} \eta\right)(Y)-\left(\mathcal{L}_{f Y} \eta\right)(X)\right\} \tilde{U} \\
& -\eta(X)(\mathcal{L} \widetilde{U} \tilde{F}) \iota_{*} Y+\eta(Y)\left(\mathcal{L}_{\tilde{U}} \tilde{F}\right) \iota_{*} X,
\end{aligned}
$$

where $\mathcal{L}$ denotes Lie differentiation and hence the following

THEOREM 3.2. Under the hypothesis of Theorem 3.1, if in addition $\tilde{M}$ is complex and $\tilde{U}$ is analytic (i. e. $\mathcal{L} \widetilde{U} \widetilde{F}=0)$, then the almost contact structure on $M$ is normal. have

If $\tilde{g}$ is a Hermitian metric on $\tilde{M}$ and $\tilde{U}$ is a unit normal to $\iota(M)$, then we

$$
\begin{aligned}
g(X, Y) & =\tilde{g}\left(\iota_{*} X, \iota_{*} Y\right) \\
& =\tilde{g}\left(\tilde{F} \iota_{*} X, \tilde{F}_{\iota_{*}} Y\right) \\
& =\tilde{g}\left(\iota_{*} f X-\eta(X) \tilde{U}, \iota_{*} f Y-\eta(Y) \tilde{U}\right) \\
& =g(f X, f Y)+\eta(X) \eta(Y) .
\end{aligned}
$$

That is, the induced metric on $M$ is an associated metric and thus $M$ possesses an almost contact metric structure.

Example. The Calabi-Eckmann manifold, $S^{2 p+1} \times S^{2 q+1}$, is a complex manifold. If we denote by $(\phi, \xi, \eta)$ the Sasakian structure of $S^{2 p+1}$ and by $\left(\phi^{\prime}, \xi^{\prime}, \eta^{\prime}\right)$ that of $S^{2 q+1}$, then the complex structure $\tilde{F}$ of $S^{2 p+1} \times S^{2 q+1}$ is given by

$$
\tilde{F}\left(X, X^{\prime}\right)=\left(\varphi X+\eta^{\prime}\left(X^{\prime}\right) \xi, \varphi^{\prime} X^{\prime}-\eta(X) \xi^{\prime}\right),
$$

where $X$ and $X^{\prime}$ are arbitrary vector fields of $S^{2 p+1}$ and $S^{2 q+1}$ respectively.

Thus we have

and

$$
\begin{aligned}
\tilde{F}(X, 0) & =\left(\varphi X,-\eta(X) \xi^{\prime}\right) \\
& =(\varphi X, 0)-\eta(X)\left(0, \xi^{\prime}\right)
\end{aligned}
$$

$$
\tilde{F}\left(0, \xi^{\prime}\right)=(\xi, 0) \text {. }
$$


$\tilde{U}=\left(0, \xi^{\prime}\right)$ being never tangent to $S^{2 p+1}$, we have that $S^{2 p+1}$ and $S^{2 q+1}$ are semiinvariant submanifolds with respect to the distinguished direction of the contact structure of the opposite factor.

\section{$\S 4$. Semi-invariant submanifolds of almost contact manifolds.}

Let $\tilde{M}$ be an almost contact manifold with $(\tilde{F} . \tilde{\xi}, \tilde{\eta})$ as the almost contact structure. Let $\iota: M \rightarrow \tilde{M}$ be an immersion such that

$$
\tilde{F}_{*} X=\iota_{*} f X+\omega(X) \tilde{\xi},
$$

$\tilde{\xi}$ is never tangent to $\iota(M)$,

for all vector fields $X$ on $M$. Then we say that $M$ is a semi-ınvariant submanifold of $\tilde{M}$.

Applying $\tilde{F}$ to the equation in (4.1) we see that $f^{2}=-I$ and $\omega(f X)=\tilde{\eta}\left(\iota_{*} X\right)$ for all $X$. Thus $f$ is an almost complex structure on $M$. If the almost contact structure is normal, then

$$
\begin{aligned}
0 & =[\tilde{F}, \tilde{F}]\left(\iota_{*} X, \iota_{*} Y\right)+d \tilde{\eta}\left(\iota_{*} X, \iota_{*} Y\right) \tilde{\xi} \\
& =\iota_{*}[f, f](X, Y)+\{d \omega(f X, Y)+d \omega(X, f Y)\} \tilde{\xi},
\end{aligned}
$$

where we have used the fact that for a normal almost contact structure $\mathcal{L}_{\xi} \tilde{F}=0$. Thus we see that the almost complex structure $f$ is integrable and $d \omega$ is of bidegree $(1,1)$, i. e., $d \omega(f X, Y)+d \omega(X, f Y)=0$ for all $X, Y$. We state this as the following theorem.

THEOREM 4.1. If $\tilde{M}$ is an almost contact manifold, then a sem-invariant submanifold $M$ possesses an induced almost complex structure. If, in addition, the almost contact structure on $\tilde{M}$ is normal, then the almost complex structure on $M$ is integrable.

Now let $\tilde{g}$ be a metric on $\tilde{M}$ associated with the almost contact structure and $g$ the induced metric on $M$. Then we have that

or

$$
\tilde{g}\left(\tilde{F} \iota_{*} X, \tilde{F} \iota_{*} Y\right)=\tilde{g}\left(\iota_{*} X, \iota_{*} Y\right)-\tilde{\eta}\left(\iota_{*} X\right) \tilde{\eta}\left(\iota_{*} Y\right)
$$

$$
g(f X, f Y)-\omega(X) \omega(Y)=g(X, Y)-\omega(f X) \omega(f Y) .
$$

Thus, if we define $\bar{g}$ by

$$
\bar{g}(X, Y)=g(X, Y)-\omega(f X) \omega(f Y),
$$

we see that $\bar{g}$ is a Hermitian metric for $f$. Here we have used the fact that $\tilde{\eta}\left(\iota_{*} X\right)=\omega(f X)$ and $\tilde{\xi}$ is not tangent to $M$.

Suppose that $\tilde{\xi}$ is normal to $M$, that is, $\tilde{g}\left(\imath_{*} X, \tilde{\xi}\right)=0$ for all $X$. Then from (4.1) we see that $\omega(X)=0$ for all $X$. That is, $M$ is an invariant submanifold of 
$\tilde{M}$, which cannot happen if $\tilde{M}$ is Sasakian, since for an invariant submanifold, we have

$$
\tilde{F}_{*} X=\iota_{*} f X \text {. }
$$

On the other hand, $\tilde{\xi}$ being a unit normal to the submanifold, we have

$$
\tilde{\nabla}_{\iota_{*} X} \tilde{\xi}=-\iota_{*} H X+\nabla_{\frac{1}{X}} \tilde{\xi},
$$

where $\tilde{\nabla}$ is the operator of covariant differentiation with respect to $\tilde{g}, H$ is the Weingarten map corresponding to $\tilde{\xi}$ and $\nabla^{\perp}$ is the connection in the normal bundle. However for a Sasakian manifold, we have $\tilde{\nabla}_{\iota_{*} X} \tilde{\xi}=\tilde{F}_{\iota_{*}} X=\iota_{*} f X$ and consequently from (4.3) we have

$$
f X=-H X,
$$

which is a contradiction since $f$ is skew-symmetric and $H$ is symmetric.

Remark. We can consider $S^{2 p} \times S^{2 q+1}$ as a hypersurface in $S^{2 p+1} \times S^{2 q+1}$. Now $S^{2 p+1} \times S^{2 q+1}$ carries an almost complex structure and hence, by a result of Tashiro [5], $S^{2 p} \times S^{2 q+1}$ possesses an almost contact structure. If $S^{2 p}$ is a semiinvariant submanifold of $S^{2 p} \times S^{2 q+1}$ then $S^{2 p}$ would be almost complex, which is not the case unless $p=1$ or 3 . Of course, $S^{2 q+1}$ cannot be a semi-invariant submanifold.

\section{§5. Semi-invariant submanifolds of manifolds with} $(\tilde{F}, \tilde{U}, \tilde{V}, \tilde{u}, \tilde{v}, \lambda)$-structure.

Let $\tilde{M}$ possess an $(\tilde{F}, \tilde{U}, \tilde{V}, \tilde{u}, \tilde{v}, \lambda)$-structure with $\{\tilde{F}, \tilde{U}, \tilde{V}, \tilde{u}, \tilde{v}, \lambda\}$ being the tensor fields of the structure. Let $\iota: M \rightarrow \tilde{M}$ be an immersion such that $\lambda\left(1-\lambda^{2}\right)$ $\neq 0$ on $\iota(M)$ and

$$
\begin{aligned}
& \tilde{F}_{\iota_{*}} X=\iota_{*} f X+\omega(X) \tilde{U}, \\
& \tilde{V}=\iota_{*} \xi, \\
& \tilde{u}\left(\iota_{*} X\right)=0,
\end{aligned}
$$

for all vector fields $X$ on $M$. Here $f, \omega, \xi$ are respectively a tensor field of type $(1,1)$, a 1 -form and a vector field on $M$. Then we say that $M$ is a semi-invariant submanifold of $\tilde{M}$ with respect to $\tilde{U}$.

If we apply $\tilde{u}$ to the first equation in (5.1) we have

$$
\lambda \tilde{v}\left({ }^{*} X\right)=\left(1-\lambda^{2}\right) \omega(X) .
$$

At a point $p$ in $M$ suppose that $\tilde{U}=\iota_{*} U$. Then we have $1-\lambda^{2}=\tilde{u}(\tilde{U})=$ $\tilde{u}\left(\iota_{*} U\right)=0$. Thus the condition $1-\lambda^{2} \neq 0$ on $M$ implies that $U$ is nowhere tangent to $M$.

Applying $\tilde{F}$ to the first equation in (5.1) we obtain

$$
-\iota_{*} X+\tilde{v}\left(\iota_{*} X\right) \tilde{V}=\iota_{*} f^{2} X+\omega(f X) \tilde{U}-\lambda \omega(X) \tilde{V} .
$$


Using (5.2) and the second equation in (5.1) this becomes

$$
f^{2} X=-X+\frac{1}{\lambda} \omega(X) \xi \text {, }
$$

$$
\omega(f X)=0 .
$$

Letting $X=\xi$ in the first equation of (5.1) we obtain

$$
\tilde{F}_{\bullet} \xi=\iota_{*} f \xi+\omega(\xi) \tilde{U},
$$

that is,

$$
f \xi=0
$$

$$
\frac{1}{\lambda} \omega(\xi)=1
$$

If we let $\eta=\frac{1}{\lambda}-\omega$ then (5.3) and (5.4) prove the following theorem.

THEOREM 5.1. If $\tilde{M}$ is a manifold with an $(\tilde{F}, \tilde{U}, \tilde{V}, \tilde{u}, \tilde{v}, \lambda)$-structure, then a semi-invariant submanifold of $\tilde{M}$ with respect to $\tilde{U}$ possesses an induced almost contact structure.

If the $(\tilde{F}, \tilde{U}, \tilde{V}, \tilde{u}, \tilde{v}, \lambda)$-structure is normal, then after some calculation similar to that in the previous section, we have that if $\lambda=$ constant on $M$,

$$
\begin{aligned}
& 0=[\tilde{F}, \tilde{F}]\left(\iota_{*} X, \iota_{*} Y\right)+d \tilde{u}\left(\iota_{*} X, \iota_{*} Y\right) \tilde{U}+d \tilde{v}\left(\iota_{*} X, \iota_{*} Y\right) \tilde{V} \\
& =\iota_{*}([f, f](X, Y)+d \eta(X, Y) \xi) \\
& +\omega(X)\left(\mathcal{L}_{\widetilde{U}} \tilde{F}\right) \iota_{*} Y-\omega(Y)(\mathcal{L} \widetilde{U} \widetilde{F}) \iota_{*} X \\
& +\left\{\left(\mathcal{L}_{f X} \omega\right)(Y)-\left(\mathcal{L}_{f Y} \omega\right)(X)\right\} \tilde{U} .
\end{aligned}
$$

Hence we have the following theorem.

THEOREM 5.2. If $\tilde{M}$ is a manifold with a normal $(\tilde{F}, \tilde{U}, \tilde{V}, \tilde{u}, \tilde{v}, \lambda)$-structure satisfying $\mathcal{L} \widetilde{U} \tilde{F}=0$, then the almost contact structure induced on a semi-invariant submanifold with respect to $\tilde{U}$ on which $\lambda=$ constant is normal.

Let $\tilde{g}$ be a metric on $\tilde{M}$ associated with the $(\tilde{F}, \tilde{U}, \tilde{V}, \tilde{u}, \tilde{v}, \lambda)$-structure. If $g$ is the metric induced on the semi-invariant submanifold $M$ then using (5.1), (5.2) and the fact that $\frac{1}{\lambda} \omega=\eta$, we have

$$
\begin{aligned}
g(f X, f Y)= & \tilde{g}\left(\iota_{*} f X, \iota_{*} f Y\right) \\
= & \tilde{g}\left(\tilde{F} \iota_{*} X-\omega(X) \tilde{U}, \tilde{F} \iota_{*} Y-\omega(Y) \tilde{U}\right) \\
= & \tilde{g}\left(\tilde{F} \iota_{*} X, \tilde{F} \iota_{*} Y\right)-\omega(X) \tilde{g}\left(\tilde{F} \iota_{*} Y, \tilde{U}\right) \\
& -\omega(Y) \tilde{g}\left(\tilde{F} \iota_{*} X, \tilde{U}\right)+\left(1-\lambda^{2}\right) \omega(X) \omega(Y)
\end{aligned}
$$


DAVID E. BLAIR, GERALD D. LUDDEN AND KENTARO YANO

$$
\begin{aligned}
= & \tilde{g}\left(\iota_{*} X, \iota_{*} Y\right)-\tilde{u}\left(\iota_{*} X\right) \tilde{u}\left(\iota_{*} Y\right)-\tilde{v}^{2}\left(\iota_{*} X\right) \tilde{v}\left(\iota_{*} Y\right) \\
& -\lambda \omega(X) \tilde{v}\left(\iota_{*} Y\right)-\lambda \omega(Y) \tilde{v}\left(\iota_{*} X\right)+\left(1-\lambda^{2}\right) \omega(X) \omega(Y) \\
= & g(X, Y)-\left(1-\lambda^{2}\right) \eta(X) \eta(Y) .
\end{aligned}
$$

If we let $\bar{g}=\frac{1}{1-\lambda^{2}} g$ then we see that $\bar{g}$ is a metric associated with the almost contact structure on $M$.

Remark 1: In the metric case $\tilde{u}\left(\iota_{*} X\right)=0$ implies that $\tilde{U}$ is normal to $M$.

Remark 2: We can consider $S^{2 p} \times S^{2 q}$ as a submanifold of condimension 2 in $E^{2 p+2 q+2}$. Now $E^{2 p+2 q+2}$ is a Kaehler manifold and so $S^{2 p} \times S^{2 q}$ carries a $(\tilde{F}, \tilde{U}, \tilde{V}, \tilde{u}, \tilde{v}, \lambda)$-structure [1]. However $S^{2 p}$ or $S^{2 q}$ cannot be semi-invariant submanifolds since they are even-dimensional.

\section{BIBLIOGRAPHY}

[1] BLAiR, D.E., G. D. LUDDEN AND K. YANO, Induced structures on submanifolds, Kōdai Math. Sem. Rep., 22 (1970), 188-198.

[2] Kubo, Y., Invariant submanifolds of codimension 2 of a manifold with $(F, G$, $u, v, \lambda)$-structure, Kōdai Math. Sem. Rep., 24 (1972), 50-61.

[3] SASAKI, S., On differentiable manifolds with certain structures which are closely related to almost contact structure, I. Tôhoku Math. J., 12 (1960), 459-476.

[4] Schouten, J.A. AND K. YANo, On invariant subspaces in the almost complex $X_{2 n}$, Ind. Math., 17 (1955), 261-269.

[5] TAshiRo, Y., On contact structures of hypersurfaces in complex manifolds, I. Tôhoku Math. J., 15 (1963), 62-78.

[6] TASHiRo, Y., On relations between the theories of almost complex spaces and almost contact spaces-Mainly on semi-invariant subspaces of almost complex spaces, Sūgaku, 16 (1964-1965), 54-61. (in Japanese).

[7] Yano, K. And M. OKumura, On $(f, g, u, v, \lambda)$-structures, Kōdai Math. Sem. Rep., 22 (1970), 401-423.

[8] Yano, K. ANd M. OKumuRa, On normal $(f, g, u, v, \lambda)$-structures on submanifolds of codimension 2 in an even-dimensional Euclidean space, Kōdai Math. Sem. Rep., 23 (1971), 172-197.

[9] YANO, K. AND M. OKUMURA, Invariant hypersurfaces of a manifold with ( $f$, $g, u, v, \lambda)$-structure, Kōdai Math. Sem. Rep., 23 (1971), 290-304.

[10] Yano, K. AND M. OKumuRA, Invariant submanifolds of a manifold with $\left(f_{\text {, }}\right.$ $g, u, v, \lambda)$-structure, Kōdai Math. Sem. Rep., 24 (1972), 75-90.

\section{Michigan State University}

